\title{
A Computational Analysis of the Effect of Mass and Radiative Heat Transfer on Free Convective Boundary Layer Flow over a Vertical Plate
}

\author{
Aiyesimi Y.M', Abah S.O., ${ }^{2, *}$, Okedayo G.T. ${ }^{3}$ \\ ${ }^{1}$ Department of Mathematics, Federal University of Technology, Minna, Nigeria \\ ${ }^{2}$ Department of Mathematics, Statistics and Computer Science, Kaduna Polytechnic, Kaduna, Nigeria \\ ${ }^{3}$ Department of Mathematical Sciences, Ondo State University of Science and Technology, Okitipupa, Nigeria \\ *Corresponding Author: brosoabah@yahoo.com
}

Copyright (C) 2013 Horizon Research Publishing All rights reserved.

\begin{abstract}
The effects of mass and radiative heat transfer on free convective flow of a viscous incompressible optically thick fluid towards a vertical surface have been investigated. The nonlinear non-dimensional, similarity-transformed boundary-layer equations governing the problem are solved using an efficient numerical method based on the Runge-Kutta integration scheme and shooting iteration technique. Numerical calculations were carried out for different values of the various non-dimensional quantities governing the flow regime. The analysis shows that the temperature decreases with increasing radiation parameter, $\mathrm{N}$ while an increase in the Prandtl number leads to a corresponding decrease in the temperature profile; a rise in the thermal Grasshof and the mass transfer number leads to increase in the velocity profile and a rise in the Schmidt number Sc leads to a decrease in the concentration profile.
\end{abstract}

Keywords Radiative Heat Transfer, Free Convective Flow, Boundary Layer

\section{Introduction}

The phenomenon of free or natural convection arises in fluids when temperature changes cause density variations leading to buoyancy forces acting on the fluid particles. Such flows which are driven by temperature differences abound in nature and have been studied extensively because of its applications in engineering, geophysical and astrophysical environments.

In general, chemical reactions in mass transfer problems include two types. A homogeneous chemical reaction which occurs uniformly throughout a given phase. The species generation in a homogeneous reaction is analogous to internal source of heat generation. On the other hand, a heterogeneous reaction takes place in a restricted region or within the boundary of a phase. It can therefore be treated as a boundary condition similar to the constant heat flux condition in heat transfer. The study of heat and mass transfer with a chemical reaction is of great practical importance to engineers and scientists because of its almost universal occurrence in many branches of science and engineering. Kandasamy et al. [2005] have studied the effects of chemical reaction and heat and mass transfer on MHD flow over a vertical stretching surface with heat source and thermal stratification.

When technological processes take place at higher temperatures thermal radiation heat transfer becomes very important and its effects cannot be neglected (Siegel $\&$ Howel, 2001). The effect of radiation on MHD flow, heat and mass transfer become more important industrially. Many processes in engineering occur at high temperatures and knowledge of radiation heat transfer becomes very important for the design of the pertinent equipment. The quality of the final product depends to a great extent on the heat controlling factors, and the knowledge of radiative heat transfer in the system can lead to a desired product with sought qualities. Different researches have been forwarded to analyze the effects of thermal radiation on different flows (Shateyi and Motsa, 2009)

Recently, there has been considerable interest in studying the problem of free convection boundary layer flow and heat transfer characteristics. The work of Ishak, Nazar and Pop(2008), investigated MHD mixed convection boundary layer flow towards a stretching vertical surface with constant wall temperature. They emphasized that, such a flow problem occurs in many industrial and technological applications which include the aerodynamic extrusion of plastic sheets cooling of metallic plates in a cooling bath, cooling of nuclear reactors, drawing, annealing and tinning of copper wires.

Hence, in the present study, we investigate the effects of mass and radiative heat transfer on free convective flow of a viscous incompressible optically thick fluid towards a vertical surface. The nonlinear nondimensional, 
similarity-transformed boundary-layer equations governing the problem are solved using an efficient numerical method based on the sixth-order Runge-Kutta integration scheme and shooting iteration technique. Numerical calculations were carried out for different values of the various non-dimensional quantities governing the flow regime.

\section{Mathematical Analysis}

A conceptual, unsteady one-dimensional laminar boundary layer flow of a viscous, incompressible, radiating fluid along a semi-infinite vertical plate in the presence of thermal and concentration buoyancy effects is considered, by taking the effect of viscous dissipation into account. The $\mathrm{x}^{\prime}$-axis is taken along the vertical infinite plate in the upward direction and the $y$-axis normal to the plate. Under these assumptions the Boussinesq's approximation for the flow field is governed by the following equations:

$$
\begin{gathered}
\frac{\partial v}{\partial y}=0 \\
\frac{\partial u}{\partial t}+v \frac{\partial u}{\partial y}=v \frac{\partial^{2} u}{\partial y^{2}}+g \beta_{T}\left(T-T_{\infty}\right)+g \beta_{C}\left(C-C_{\infty}\right) \\
\frac{\partial T}{\partial t}+v \frac{\partial T}{\partial y}=\alpha \frac{\partial^{2} T}{\partial y^{2}}-\frac{1}{\rho C_{p}} \frac{\partial q}{\partial y} \\
\frac{\partial C}{\partial t}+v \frac{\partial C}{\partial y}=D \frac{\partial^{2} C}{\partial y^{2}}+\lambda\left(C-C_{\infty}\right)
\end{gathered}
$$

With the following boundary conditions,

$$
\begin{gathered}
u=U, T=T_{w}, C=C_{w} \text {, at } y=0 \\
u \rightarrow 0, T \rightarrow T_{\infty}, C \rightarrow C_{\infty}, \text { as } y \rightarrow \infty \text { for } t>0
\end{gathered}
$$

In order to write the governing equations and the boundary conditions in dimensionless form, the following nondimensional quantities are introduced:

$$
\begin{gathered}
\frac{u}{u_{\infty}}=f(\eta) \text {, where } \eta=\frac{y}{2 \sqrt{v t}}, \theta(\eta)=\frac{T-T_{\infty}}{T_{w}-T_{\infty}}, \\
\phi(\eta)=\frac{C-C_{\infty}}{C_{w}-C_{\infty}} \\
\frac{4 \operatorname{tg}}{u} \beta_{T}\left(T_{w}-T_{\infty}\right)=G_{T} \\
\frac{4 t g}{u} \beta_{C}\left(C_{w}-C_{\infty}\right)=G_{C} \\
\frac{16 T_{\infty}^{3} \sigma_{f}}{3 v k_{f}}=\frac{1}{N}, \operatorname{Pr}=\frac{v}{\alpha}, \\
-c=V_{o} \sqrt{\frac{t}{v}}, \quad S c=\frac{v}{D} \quad \text { and } \quad 4 \lambda t=\tau
\end{gathered}
$$

$$
q=\text { Local radiation heat flux }
$$

But, $q=-\frac{4 \sigma_{f}}{3 k_{f}} \frac{\partial T^{4}}{\partial y}$ this is Rosseland approximation (Brewster 1992)where $\sigma_{f}$ is the Stefan-Boltzmanconstant and $k_{f}$ is the absorption coefficient.

It should be noted that by using the Rosseland approximation the present analysis is limited to optically thick fluids.

Therefore from equation (2.3)

$$
\frac{\partial q}{\partial y}=-\frac{4 \sigma_{f}}{3 k_{f}} \frac{\partial^{2} T^{4}}{\partial y^{2}}
$$

But, $T^{4}=4 T_{\infty}^{3} T-3 T_{\infty}^{4}$ by Taylors' expansion

$$
\therefore \frac{\partial q}{\partial y}=-\frac{16}{3} \frac{T_{\infty}^{3} \sigma_{f}}{k_{f}} \frac{\partial^{2} T}{\partial y^{2}}
$$

In view of the above dimensionless quantities, the governing equations are reduced to the following dimensionless form:

$$
\begin{gathered}
f^{\prime \prime}(\eta)+2(\eta+c) f^{\prime}(\eta)+G_{T} \theta(\eta)+G_{C} \phi(\eta)=0 \\
\theta^{\prime \prime}(\eta)+\left[\frac{2 N \operatorname{Pr}}{N+\operatorname{Pr}}\right](\eta+c) \theta^{\prime}(\eta)=0 \\
\phi^{\prime \prime}(\eta)+2 S c(\eta+c) \phi^{\prime}(\eta)+S c \tau \phi(\eta)=0 \\
f(0)=\theta(0)=\phi(0)=1 \\
f(\infty)=\theta(\infty)=\phi(\infty)=0
\end{gathered}
$$

\section{Method of Solution}

The system in (2.6) through (2.10) has been solved numerically via Nachtseim and Swigertshooting iteration technique together with the Runge-Kuttasixthorder integration scheme. The numerical computations were carried out using a program which uses a symbolic and computational computer language MAPLE (Heck, 2003), the software package used is Maple 14. The physical parameters of interest such as the skinfriction coefficient, the Nusselt and Sherwood Numbers which are respectively proportional to $-f^{\prime}(0),-\theta^{\prime}(0)$ and $-\phi^{\prime}(0)$ have been numerically determined and presented in the table 1 and also graphically below.

\section{Results and Discussion}

The numerical simulation depicted by the table and the figures $1-9$ shows that skin friction decreases with increasing thermal Grashof number when the Nusselt and 
Sherwood numbers are kept constant. The same phenomenon is observed with variation in the SolutalGrashof number. Increasing the magnitude of Prandtl, Schidmt and radiation numbers, results in a corresponding increase in the skin friction. It is also observed that velocity varies directly with both thermal and mass Grashof numbers. Figure 3 demonstrates the effect of the radiation parameter on the temperature profile showing an inverse relation. The effect of the unsteadiness parameter on the velocity, temperature and concentration profile is demonstrated in figures $4-6$ which varies inversely with the parameter. Figure 7 shows that the temperature decreases with increase in the Prandtl number. Figure 8 show that the concentration varies inversely with the Schidmt number. Finally, Figure 9 demonstrates the direct variation of the concentration profile with rate of chemical reaction.

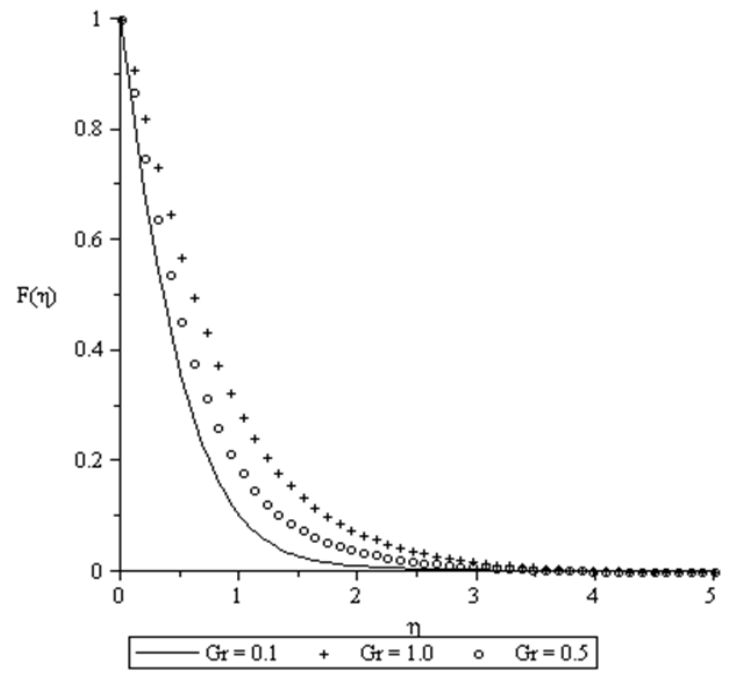

Figure 1. Velocity profiles for various values of $\mathrm{Gr}$ when $\mathrm{Gc}=0.1, \mathrm{~N}=0.1$, $\mathrm{c}=0.5, \operatorname{Pr}=0.72, \mathrm{Sc}=0.24, \tau=0.1$.

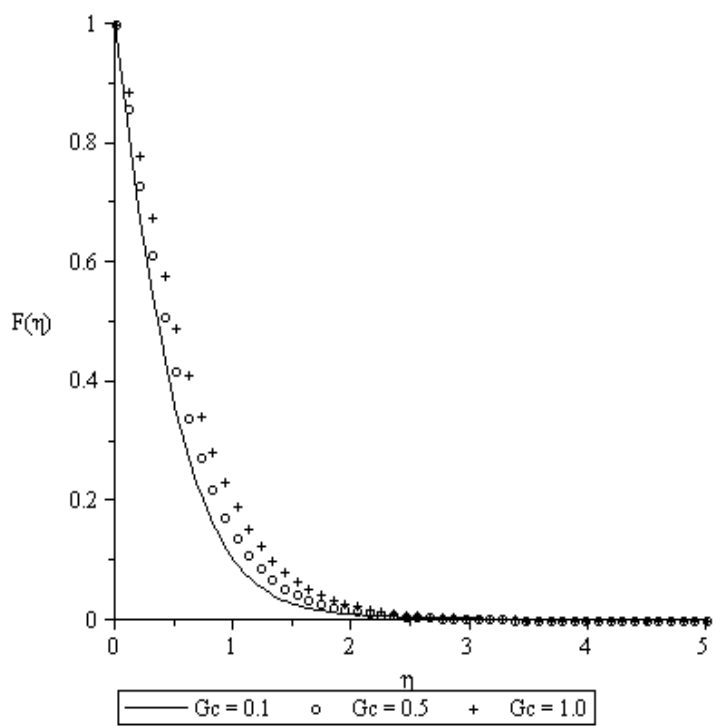

Figure2. Velocity profiles for various values of $\mathrm{Gc}$ when $\mathrm{Gr}=0.1, \mathrm{~N}=0.1$, $\mathrm{c}=0.5, \operatorname{Pr}=0.72, \mathrm{Sc}=0.24, \tau=0.1$.

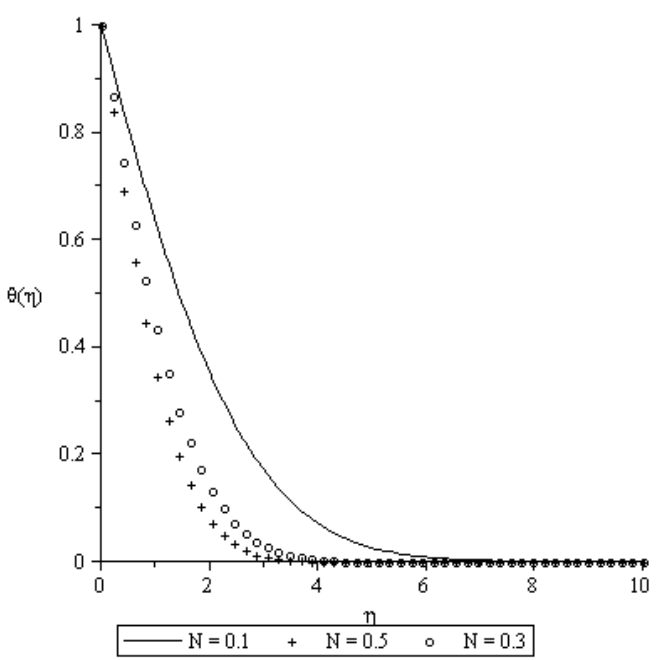

Figure 3. Temperature profiles for various values of radiation parameter $\mathrm{N}$ when $\mathrm{Gr}=0.1, \mathrm{Gc}=0.1, \mathrm{c}=0.5, \operatorname{Pr}=0.72, \mathrm{Sc}=0.24, \tau=0.1$

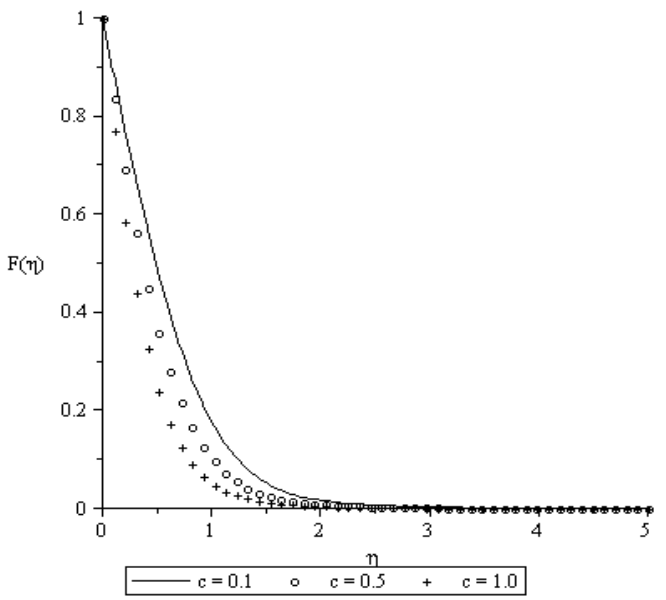

Figure 4. Velocity profiles for various value of $\mathrm{c}$ when $\mathrm{Gr}=0.1, \mathrm{Gc}=0.1$, $\mathrm{N}=0.1, \mathrm{Pr}=0.72, \mathrm{Sc}=0.24, \tau=0.1$

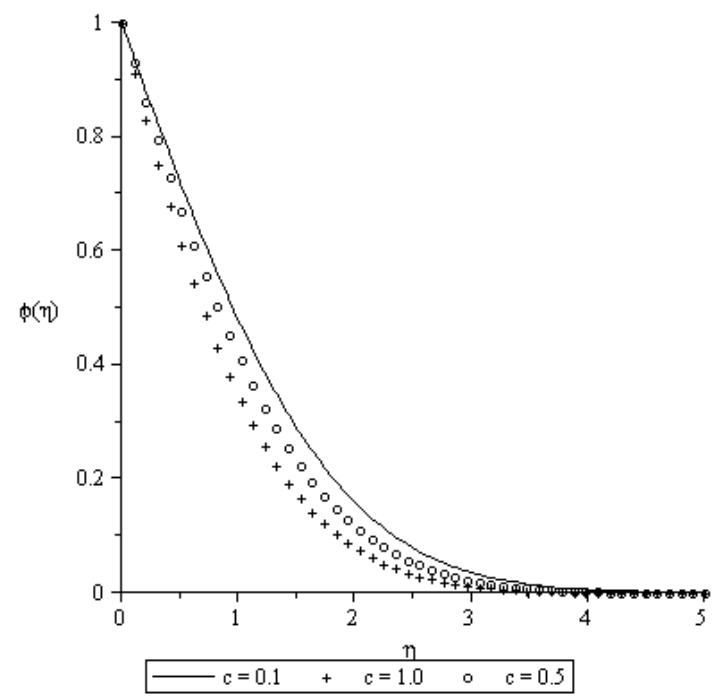

Figure 5. Concentration profiles for various value of $\mathrm{c}$ when $\mathrm{Gr}=0.1, \mathrm{Gc}$ $=0.1, \mathrm{~N}=0.1, \mathrm{Pr}=0.72, \mathrm{Sc}=0.24, \tau=0.1$. 


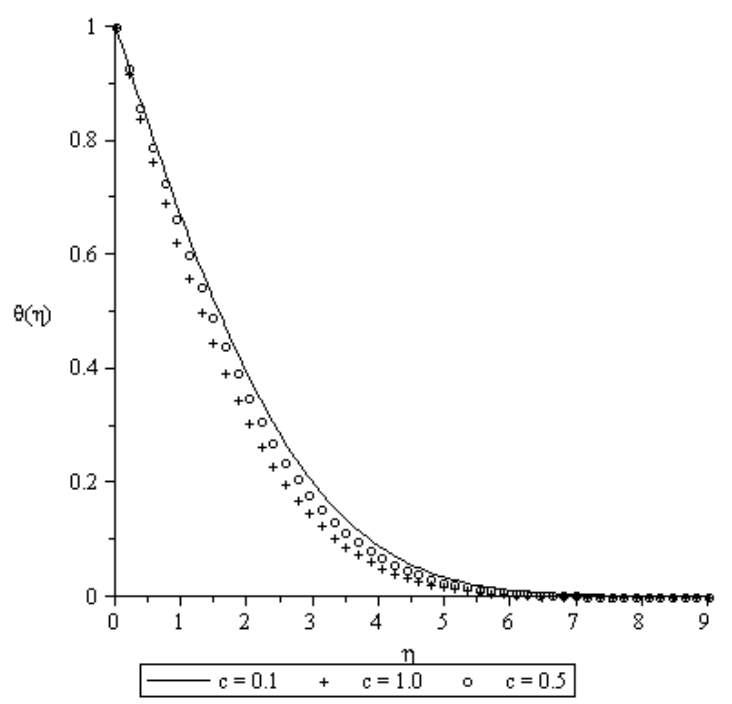

Figure 6. Temperature profiles for various value of $\mathrm{c}$ when $\mathrm{Gr}=0.1, \mathrm{Gc}=$ $0.1, \mathrm{~N}=0.1, \mathrm{Pr}=0.72, \mathrm{Sc}=0.24, \tau=0.1$.

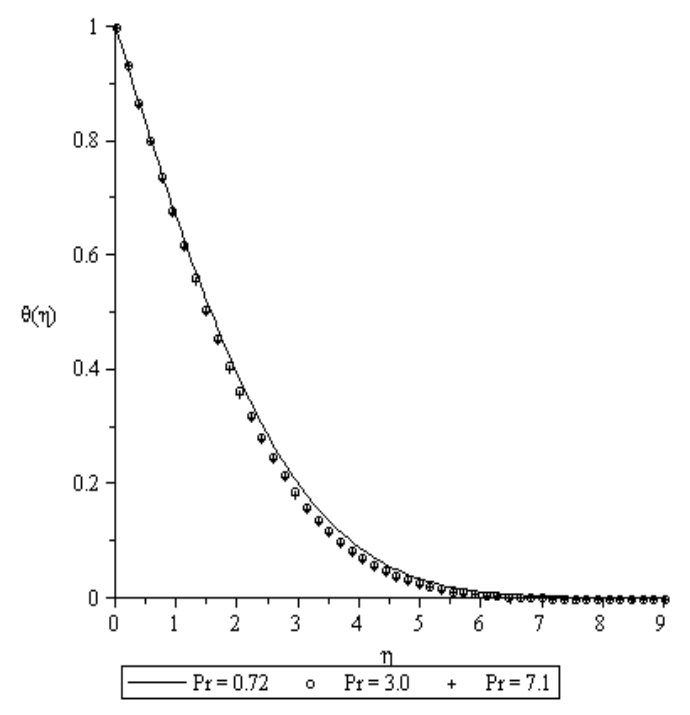

Figure 7. Temperature profiles for various values of Prandtl number Pr when $\mathrm{Gr}=0.1, \mathrm{Gc}=0.1, \mathrm{~N}=0.1, \mathrm{c}=0.5, \mathrm{Sc}=0.24, \tau=0.1$.

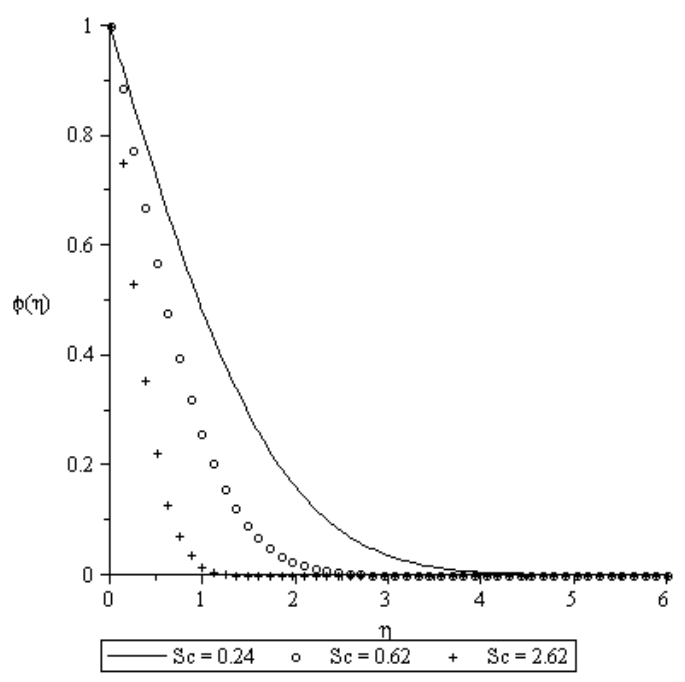

Figure 8. Concentration profiles for various values of Schmidt number $\mathrm{Sc}$ when $\mathrm{Gr}=0.1, \mathrm{Gc}=0.1, \mathrm{~N}=0.1, \mathrm{c}=0.5, \mathrm{Pr}=0.72, \tau=0.1$.

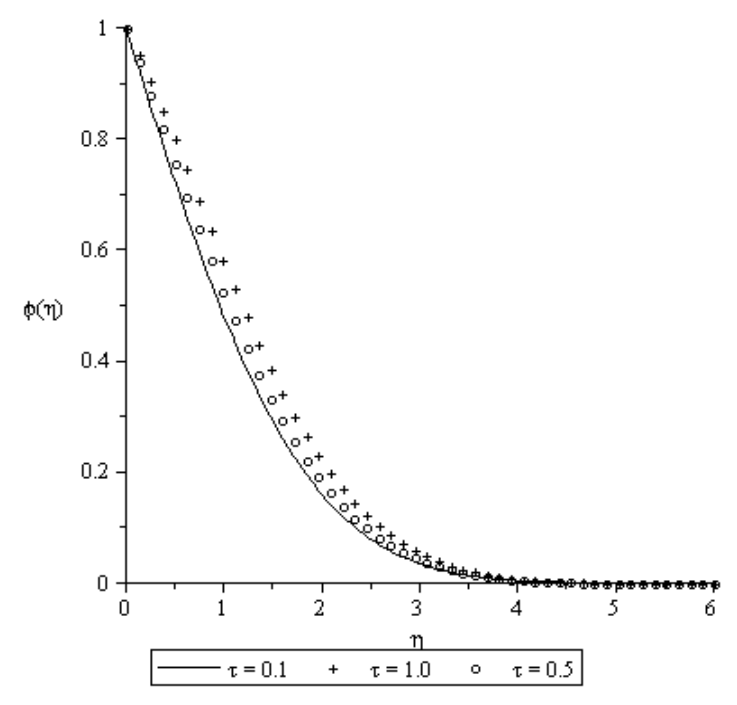

Figure 9. Concentration profiles for various values of chemical reaction parameter $\tau$ whenGr $=0.1, \mathrm{Gc}=0.1, \mathrm{~N}=0.1, \mathrm{c}=0.5, \mathrm{Pr}=0.72, \mathrm{Sc}=0.24$. 
Table 1. Numerical computations showing $f^{\prime}(0), \theta^{\prime}(0)$ and $\phi^{\prime}(0)$ for various embedded flow parameters

\begin{tabular}{|c|c|c|c|c|c|c|c|c|c|}
\hline $\mathrm{Gr}$ & $\mathrm{Gc}$ & $\mathrm{a}$ & $\mathrm{Pr}$ & $\mathrm{N}$ & $\mathrm{Sc}$ & $\tau$ & $-f^{\prime}(0)$ & $-\theta^{\prime}(0)$ & $-\phi^{\prime}(0)$ \\
\hline 0.1 & 0.1 & 0.1 & 0.72 & 0.1 & 0.24 & 1 & 1.1017225079 & 0.3456276073 & 0.366483153 \\
\hline 0.5 & 0.1 & 0.1 & 0.72 & 0.1 & 0.24 & 1 & 0.7603393511 & 0.3456276073 & 0.366483153 \\
\hline 1.0 & 0.1 & 0.1 & 0.72 & 0.1 & 0.24 & 1 & 0.3336104051 & 0.3456276073 & 0.366483153 \\
\hline 0.1 & 0.5 & 0.1 & 0.72 & 0.1 & 0.24 & 1 & 0.8151565808 & 0.3456276073 & 0.366483153 \\
\hline 0.1 & 1.0 & 0.1 & 0.72 & 0.1 & 0.24 & 1 & 0.4569491718 & 0.3456276073 & 0.366483153 \\
\hline 0.1 & 0.1 & 0.2 & 0.72 & 0.1 & 0.24 & 1 & 1.2357556660 & 0.3570428659 & 0.402490401 \\
\hline 0.1 & 0.1 & 0.3 & 0.72 & 0.1 & 0.24 & 1 & 1.3754144122 & 0.3686115810 & 0.439142011 \\
\hline 0.1 & 0.1 & 0.1 & 3.0 & 0.1 & 0.24 & 1 & 1.1039978234 & 0.3634395174 & 0.366483153 \\
\hline 0.1 & 0.1 & 0.1 & 7.1 & 0.1 & 0.24 & 1 & 1.1044346872 & 0.3669912648 & 0.366483153 \\
\hline 0.1 & 0.1 & 0.1 & 0.72 & 0.3 & 0.24 & 1 & 1.1212745534 & 0.5465161312 & 0.366483153 \\
\hline 0.1 & 0.1 & 0.1 & 0.72 & 0.6 & 0.24 & 1 & 1.1299943107 & 0.6877591023 & 0.366483153 \\
\hline 0.1 & 0.1 & 0.1 & 0.72 & 0.1 & 0.62 & 1 & 1.1213656634 & 0.3456276073 & 0.624057431 \\
\hline 0.1 & 0.1 & 0.1 & 0.72 & 0.1 & 0.78 & 1 & 1.1256056135 & 0.3456276073 & 0.712703275 \\
\hline 0.1 & 0.1 & 0.1 & 0.72 & 0.1 & 0.24 & 2 & 1.0869399111 & 0.3456276073 & 0.048000000 \\
\hline 0.1 & 0.1 & 0.1 & 0.72 & 0.1 & 0.24 & 3 & 1.0497691195 & 0.3456276073 & 0.606873211 \\
\hline
\end{tabular}

\section{Conclusions}

A theoretical study of the boundary layer momentum heat and mass transfer characteristics radiative incompressible viscous steady boundary layer flow towards a vertical surface has been carried out. The nonlinear boundary layer equations governing the problem under consideration are solved numerically by applying an efficient numerical technique based on Runge-Kutta of order six as well as the shooting method.Numerical experiments showing the influence of the variousphysical parameters on the velocity, temperature and concentration profile were performed.

The present analysis has shown that:

- As the radiation parameter, $\mathrm{N}$, increases the temperature decreases.

- An increase in the Prandtl number leads to a decrease in the temperature profile.

- A rise in the thermal Grasshof and the mass transfer number leads to increase in the velocity profile.

- A rise in the Schmidt number Sc leads to a decrease in the concentration profile.

\section{REFERENCES}

[1] Aziz,A. A Similarity solution for laminar thermal boundary layer over a flat plate with a convective surface boundary condition, Commum Nonlinear SciNumerSimulat 14 1064-1068, 2009.
[2] Brewster M. Q, Thermal radiative transfer and properties, John Wiley \& Sons, Inc., Newyork, 1992.

[3] Fang, T. Similarity solutions for a moving-flat plate thermal boundary layer, Acta Mech. 163 161-172, 2003.

[4] Heck, A. Introduction to Maple, 3rd Edition, Springer-Verlag, 2003.

[5] Hossain, M.A., H.S. Takhar, Radiation effects on mixed convection along a vertical plate with uniform surface temperature, Heat Mass Transfer 31 243-248, 1996.

[6] Ishak A, Nazar R. and Pop I. Hydromagnetic Flow and Heat Transfer Adjacent To a Stretching Vertical Sheet, Heat and Mass Transfer, 44, 921-927, 2008.

[7] Kandasamy, A., Periasamy, K. and SivagnanaPrabhu, K.K. Chemical reaction, heat and mass transfer on MHD flow over a vertical stretching surface with heat source and thermal stratification effects, Int. J. Heat Mass Tran., 48, 4557-4561, 2005.

[8] Makinde O. D., Olanrewaju P. O., Buoyancy effects on thermal boundary layer over a vertical plate with a convective surface boundary condition, Transactions ASME Journal of Fluids Engineering, Vol. 132, 044502(1-4), 2010.

[9] Sakiadis,B.C. Boundary-layer behavior on continuous solid surfaces: I. Boundary-layer equations for two-dimensional and axisymmetric flow, AIChE J. 7 26-28, 1961.

[10] Shateyi, S., \&Motsa, S.S. (2009). Thermal Radiation Effects on Heat and Mass Transfer over an Unsteady Stretching Surface. Mathematical Problems in Engineering, Vol. 2009, Article ID 965603, 13 pages doi:10.1155/2009/965603.

[11] Siegel, R., \& Howell, J.R. (2001). Thermal Radiation Heat 
Transfer, Speedy Hen, London, CA, United Kingdom, (ISBN: 1560328398 / 1-56032-839-8).

[12] Tsou, F.K., E.M. Sparrow, R.J. Glodstein, Flow and heat transfer in the boundary layer on a continuous moving surface, Int. J. Heat Mass Transfer 10 219-235, 1967.

[13] Weyl, H. On the differential equations of the simplest boundary-layer problem, Ann. Math. 43 381-407, 1942. 